\title{
Wavelets and Waves in Optical Signal Preprocessing
}

\author{
Th. Beth, A. Klappenecker, M. Schmid and D. Zerfowski
}

Institut für Algorithmen and Kognitive Systeme, Universität Karlsruhe, 76128 Karlsruhe, Germany

\section{Introduction}

Wavelets are versatile tools in signal analysis and representation, complementing existing tools from harmonic analysis. In recent years, an upsurge of interest in wavelet methods influenced the area of image processing. Wavelet techniques combine traditional methods from imaging and harmonic analysis, thus yealding powerful and efficient algorithms for various applications.

Using wavelet transforms it is possible to build highly reliable compression schemes for pictures, providing bandwidth economic preview and browsing schemes. Taking the underlying semantics of the signal into account, a comprehensive description of the signal can also be obtained by an adapted coding of the occurring elementary features or objects. Using this semantics leads to a high level description of the signal. For this reason an image preprocessing step, a feature extraction has to be involved, which can again be achieved by using wavelet methods, e.g. for edge detection and segmentation. In this paper we discuss wavelet based feature extraction methods and we describe an optical implementation using diffractive elements.

\section{Wavelets}

We briefly recall some basic notions from wavelet analysis. The elementary building blocks of wavelet analysis are obtained by translations and unitary dilations from a single square integrable function $\psi$ - the wavelet. A square integrable signal $s \in L^{2}(\mathbb{R})$ can then be analysed by the scalar products

$$
s \Leftrightarrow\left\langle\frac{1}{\sqrt{a}} \psi\left(\frac{\cdot \Leftrightarrow b}{a}\right) \mid s\right\rangle, \quad a, b \in \mathbb{R}, a>0 .
$$

The wavelet $\psi$ is usually assumed to be a function that is sufficiently localized in time as well as in frequency and has some vanishing moments. 
A typical example for a wavelet is given by the mexican hat function $\psi(x)=\left(x^{2} \Leftrightarrow 1\right) e^{-x^{2} / 2}$.

The continuous wavelet transform (1) can be generalized to higher dimensions where we have in addition to the dilation and translation operations a rotation operation. Clearly, the rotation is superfluous, if rotation symmetric wavelets are use. For example, a rotation symmetric $2 \mathrm{D}$ mexican hat function is given by $\left(\|x\|_{\mathrm{R}^{2}}^{2} \Leftrightarrow 2\right) \cdot \exp \left(\Leftrightarrow\|x\|_{\mathrm{R}^{2}}^{2} / 2\right)$ We will use this wavelet later in an optical implementation for edge detection.

The continuous wavelet transform (1) is rarely used in applications because it leads in general to a highly redundant representation of the signal that is rather expensive from a computational point of view. Typically, only a part of the wavelet coefficients is necessary for feature detection. For example, in edge detection applications only a few scales have to be considered.

Compression applications lead to a natural interest in non-redundant representations of a signal. The most compact way is to express the signal with respect to a basis of the Hilbert space $L^{2}$. A particularly nice class of wavelets leads to biorthogonal wavelet bases of the Hilbert space $L^{2}(\mathbb{R})$. A biorthogonal wavelet basis is given by a Riesz basis of the form $\psi_{j, k}(x)=2^{-j / 2} \psi\left(2^{-j} x \Leftrightarrow k\right)$, with $j, k \in \mathbb{Z}$, that has a dual basis ${ }^{1} \tilde{\psi}_{j, k}$ of the same form. A pair of biorthogonal wavelet bases allows the representation of a signal as follows:

$$
s=\sum_{j, k \in \mathbb{Z}}\left\langle\widetilde{\psi}_{j, k} \mid s\right\rangle \psi_{j, k}=\sum_{j, k \in \mathbb{Z}}\left\langle\psi_{j, k} \mid s\right\rangle \widetilde{\psi}_{j, k} .
$$

\section{Compression}

Radiologic image data in the order of at least several peta bytes comes up every year. Clearly, there is a natural demand for effective compression methods, since archiving of radiologic images over several years is legally obliged. Wavelets can be used successfully in radiologic image compression applications. We sketch the main ideas of a wavelet based compression method in this section.

The basic principle of a wavelet based compression scheme can be subdivided into three major steps: the input signal is transformed in order to decorrelate adjacent signal samples, then the entropy of the resulting coefficients is reduced by quantization. In a final step the redundancy is removed by passing the quantized coefficients through an entropy coder.

${ }_{1}$ A Riesz basis of a Hilbert space $H$ is a family of linear independent vectors $v_{i}, i \in I$, that constitute a frame. The dual basis is defined as usual by $\left\langle\psi_{j, k} \mid \widetilde{\psi}_{l, m}\right\rangle=\delta_{j, l} \delta_{k, m}$. 
An implementation of this scheme should be as fast as possible. If we use scalar quantization, i. e. a quantization operation that can be applied independently to each coefficient, then the quantization has linear complexity. The entropy coding can for example be done by arithmetic coding. A method with particular high throughput was developed recently at the authors' institution [9]. It remains to give a fast algorithm for the wavelet transform.

All well-behaved biorthogonal wavelet bases can be constructed with the help of multiresolution analyses. For compactly supported biorthogonal wavelets this construction leads directly to a fast algorithm for the discrete wavelet transform. For the ease of exposition we leave out some details that can be found for example in [8] and mainly focus on the points that are relevant for our application.

A multiresolution analysis of $L^{2}(\mathbb{R})$ is a sequence of nested closed subspaces $V_{j} \subset V_{j-1}$ of $L^{2}(\mathbb{R})$ such that $\bigcap_{j \in \mathbb{Z}} V_{j}=\{0\}$ and $\bigcup_{j \in \mathbb{Z}} V_{j}$ is dense in $L^{2}$. Moreover, the subspaces are linked by $f(x) \in V_{j} \Leftrightarrow f(2 x) \in$ $V_{j-1}$ and the subspace $V_{0}$ is supposed to have a Riesz basis of the form $\varphi(x \Leftrightarrow k), k \in \mathbb{Z}$. The function $\varphi$ is called scaling function. We use again the convenient abbreviation $\varphi_{j, k}(x)$ to denote the dilated and translated functions $2^{-j / 2} \varphi\left(2^{-j} x \Leftrightarrow k\right)$. The scaling function manifests the connection between two different scales through the following formula: $\varphi(x / 2)=\sum_{n \in \mathbb{Z}} h_{n} \sqrt{2} \varphi(x \Leftrightarrow n)$, which can be written in Fourier space as $\hat{\varphi}(2 \omega)=m_{0}(\omega) \hat{\varphi}(\omega)$, with $m_{0}(\omega)=2^{-1 / 2} \sum h_{n} e^{-i n \omega}$. We call $m_{0}(\omega)$ the scaling filter associated with $\varphi$.

A pair of multiresolution analyses $V_{j}$ and $\widetilde{V_{j}}$ of $L^{2}(\mathbb{R})$ is called biorthogonal with compactly supported dual scaling functions iff the scaling functions $\varphi, \tilde{\varphi}$ are dual in the sense that $\langle\varphi(x) \mid \tilde{\varphi}(x \Leftrightarrow k)\rangle=\delta_{k, 0}$ holds and $\varphi$ and $\tilde{\varphi}$ are compactly supported functions. Such a pair of multiresolution analyses allows us to define the functions

$$
\widehat{\psi}(2 \omega)=e^{-i \omega} \overline{m_{0}(\omega+\pi)} \hat{\varphi}(\omega), \quad \hat{\widetilde{\psi}}(2 \omega)=e^{-i \omega} \overline{\tilde{m}_{0}(\omega+\pi)} \hat{\varphi}(\omega),
$$

where we used $\hat{f}$ to denote the Fourier transform of a function $f ; m_{0}, \widetilde{m}_{0}$ denote the scaling filters associated to $\varphi, \tilde{\varphi}$. It turns out that $\psi_{j, k}, j, k \in \mathbb{Z}$, is a Riesz basis of $L^{2}(\mathbb{R})$ with dual $\hat{\psi}_{j, k}$, see [6] for a proof. Thus, we can construct biorthogonal wavelet bases from multiresolution analyses.

The structure of the multiresolution analysis allows in a natural way to define certain approximations to the signal. For all $s \in L^{2}(\mathbb{R})$ a projection on $V_{j}$ in the direction of $\tilde{V}_{j}^{\perp}$ is given by

$$
P_{j} s=\sum_{k \in \mathbb{Z}}\left\langle\tilde{\varphi}_{j, k} \mid s\right\rangle \varphi_{j, k}
$$


The details missing in a coarse approximation can be described by wavelets; this is the key idea of Mallat's fast wavelet transform algorithm. Given a fine approximation of a signal $s$, say in terms of the coefficients $s_{j-1, k}=\left\langle\tilde{\varphi}_{j-1, k} \mid s\right\rangle, k \in \mathrm{Z}$, then it is possible to calculate efficiently the coefficients $s_{j, k}=\left\langle\tilde{\varphi}_{j, k} \mid s\right\rangle$ and $d_{j, k}=\left\langle\tilde{\psi}_{j, k} \mid s\right\rangle$. In fact, the corresponding operations can be realized by a 2-channel perfect reconstruction filter bank. Similarly, we can again calculate efficiently the coefficients $s_{j+1, k}$ and $d_{j+1, k}$ from the coefficients $s_{j, k}$. The dataflow of Mallat's algorithm is shown in Figure 1.

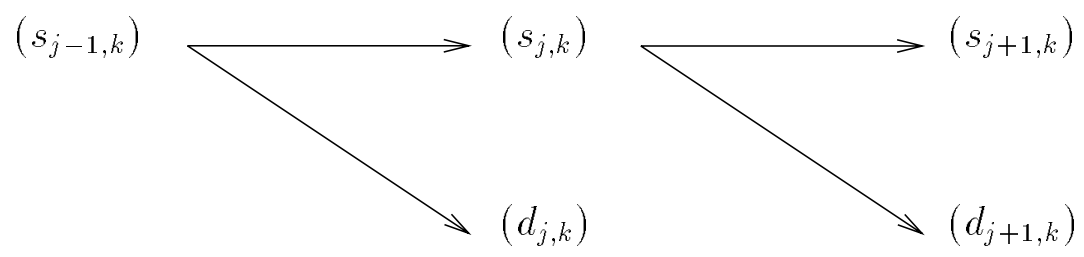

Figure 1. Dataflow of Mallat's fast wavelet transform algorithm.

This algorithm can be extended without any difficulty to higher dimensions using tensor products. It is very convenient from a practical point of view that smooth signals give wavelet coefficients $d_{j, k}$ near zero, provided the wavelet is smooth enough. Hence, after the quantization a large part of the wavelet coefficients is zero, so that for a proper choice of the wavelet we obtain a compact description of the signal. The results of such a compression scheme are exemplified in Figure 2 for a medical image. For the same compression ratio, the JPEG compression standard leads to significantly higher degradation of the image. Wavelet compression is in terms of peak-signal-to-noise-ratio $2 \mathrm{~dB}$ better.

\section{Optical Signal Preprocessing}

In recent image processing applications large amount of data has to be manipulated under hard time constraints, especially in real time or medical applications. Fast digital algorithms for signal preprocessing have been developed and proposed but for high resolution applications the needed performance has not been reached yet. For this reason alternative highly parallel methods like optical signal processing seems to be a promising new way. Nowadays optical preprocessing becomes in range of 

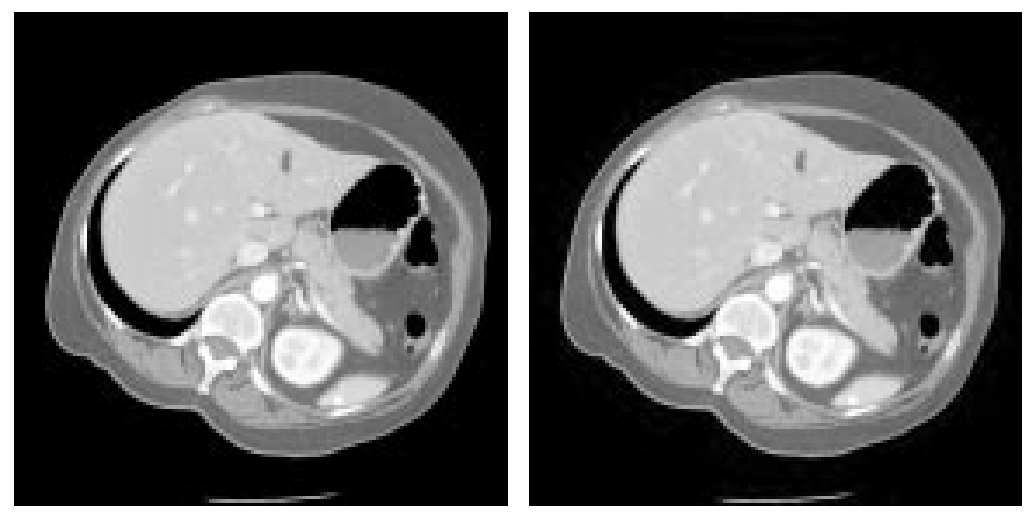

Figure 2. Computer tomographic image of a human body. Left: original image. Right: wavelet compressed image with rate 17.8:1.

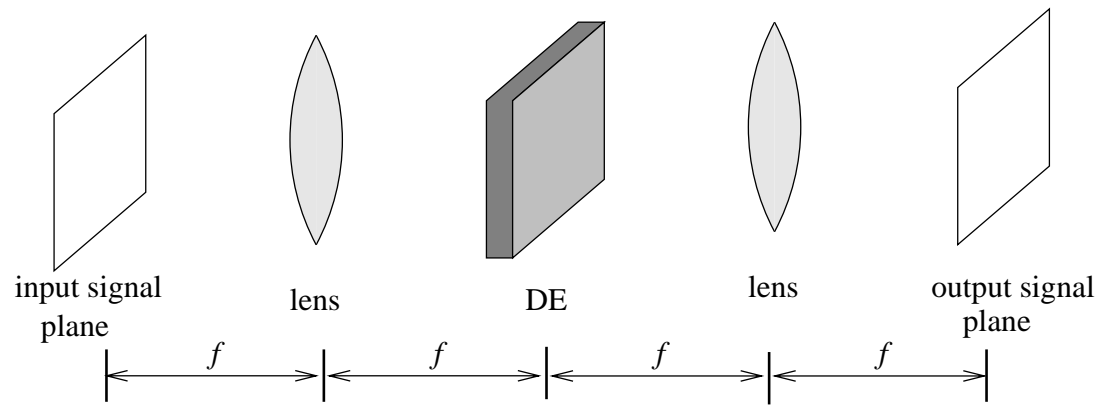

Figure 3. $4 f$-setup

current technology due to fast and high resolution spatial light modulators (SLM), which are already commercially available.

In principle every linear transform in $L^{2}\left(\mathbb{R}^{2}\right)$ can be implemented by an optical setup [1]. The input, a complex two dimensional signal, is represented as a wave front on which the optical system operates at highest possible parallelism. Convolutional operators are of special interest in signal preprocessing. These operators can be build in an optical system by the well-known $4 f$-setup, which is based on the convolutional theorem of the Fourier transform. A schematic view of a $4 f$-setup is given in Figure 3. A monochromatic, coherent, two dimensional input signal from the left is propagated through a lens with focus $f$, performing the Fourier transform. In the Fourier plane at the distance $2 f$ a pre-calculated diffractive element (DE) performs the pointwise multiplication in the frequency space and can be viewed as an adaptable filter. There are several types 
of diffractive elements, which modulate the amplitude and/or phase of an incoming wavefront. The second lens on the right realizes a second Fourier transform.

Due to the practical restrictions of manufacturing of DEs only phase or amplitude modulating elements can be produced. Because of the light efficiency phase-only elements are preferred.

In general the kernel of the intendend convolution is a complex valued function with non-constant amplitude and thus can not be realized by a phase-only DE. A method to overcome this problem is to search for a phase-only DE with the same impulse response in a given detection area (or output signal window). Because the impulse response outside of the signal window can freely be chosen, we gain some parameters of freedom for the design of the DE.

Given an impulse response $h_{0}(x, y)$ in the domain $S$ (the signal window) we search for a bandlimited signal $h(x, y)$ which is equal to $h_{0}$ in $S$ and satisfies the conditions

$$
\left(\mathcal{F}^{-1} h\right)(u, v)= \begin{cases}e^{i \varphi(u, v)} & \text { if }(u, v) \in D \\ 0 & \text { else, }\end{cases}
$$

where $D$ describes the domain of the diffractive element. The calculation of $h(x, y)$ is an ill-posed problem in the sense of Hadamard, because in general all conditions for well-posed problems can be violated [3]. In spite of the freedom outside the signal window normally no solution exists and therefore we are interested in a good approximation. Furthermore, for a given impulse response inside the signal window there might exist several diffractive elements, where the impulse responses differ only outside the signal window.

After this preparation we are ready to give an iterative algorithm based on generalized projections for calculating phase-only DEs for the desired filter transfer function. One special method is the so-called IFTA algorithm [10] (Iterative Fourier Transform Algorithm) which is a slight modification of the Gerchberg-Saxton Algorithm [4] for DEs. The main idea of these algorithms is the following: We consider one arbitrary initial point $f_{0}$ and two convex sets $M_{1}, M_{2}, M_{c}:=M_{1} \cap M_{2} \neq \emptyset$ in a Hilbert space, with two projections $\mathcal{P}_{1}, \mathcal{P}_{2}$ onto the two sets. Applying one projection on the initial point, it is mapped into the corresponding set. In the sequel the alternating use of both projection converges to some point in the intersection $M_{c}$. Thus $f_{n+1}=\mathcal{P}_{1} f_{n}$ and $f_{n+2}=\mathcal{P}_{2} f_{n+1}$ imply

$$
\lim _{n \rightarrow \infty}\left\|\mathcal{P}_{c} f_{n} \Leftrightarrow f_{n}\right\|=0
$$


for the unknown projection $\mathcal{P}_{c}$ on $M_{c}[5]$.

In our special application the set of all desired impulse responses with the fixed signal window and the set of the Fourier transformed phaseonly elements represent the two sets $M_{1}, M_{2}$. The projection operators $\mathcal{P}_{1}$ and $\mathcal{P}_{2}$ are given by

$$
\begin{aligned}
\left(\mathcal{P}_{1} g\right)(x, y) & := \begin{cases}h_{0}(x, y) & \text { if }(x, y) \in S \\
g(x, y) & \text { else, }\end{cases} \\
\mathcal{P}_{2} & :=\mathcal{F}_{\mathcal{P}^{\prime}{ }_{2} \mathcal{F}^{-1}} \text { with } \\
\left(\mathcal{P}^{\prime}{ }_{2} \hat{h}\right)(u, v) & := \begin{cases}\frac{\hat{h}(u, v)}{|\widehat{h}(u, v)|} & \text { if } \hat{h}(u, v) \neq 0 \\
1 & \text { else. }\end{cases}
\end{aligned}
$$

At this point we have to mention that in this special case the set $M_{1}$ is not convex and we even can not guarantee that the intersection $M_{c}$ is not empty. For this reason we have to consider a generalized version to non convex sets [7], for which the convergence can not be guaranteed, too. This lack of convergence is partially compensated by the use of the square distance error, which can be minimized in the iteration process. For the projections

$$
h_{n}=\mathcal{P}_{1} g_{n} \quad \text { and } \quad g_{n+1}=\mathcal{P}_{2} h_{n}
$$

we use the squared distance errors $S D E_{M_{i}}(f):=\left\|f \Leftrightarrow \mathcal{P}_{i} f\right\|, i=1,2$ related to $M_{1}$ and $M_{2}$ and it holds

$$
S D E_{M_{1}}\left(g_{n+1}\right) \leq S D E_{M_{1}}\left(g_{n}\right) \text { and } S D E_{M_{2}}\left(h_{n+1}\right) \leq S D E_{M_{2}}\left(h_{n}\right) .
$$

At this point of our discussion we posses an iterative tool to calculate a phase-only DE for a desired convolutional filter.

We now consider implementations of filter functions, which promise great advantages, e.g., in the area of medical imaging: the optical wavelet transforms. One main advantage of using wavelet transforms in image preprocessing is their signal analysing properties at different scales and orientations. For example, different kinds of vertices, sharp or smooth ones, can be detected in different scales. With the knowledge of this kind of information succeeding image processing steps like feature extraction can be made more efficient.

In the previously described design process of the DEs was restricted to one filter function, but for a multiresolution analysis a family of wavelets has to be used. In the optical implementation we overcome this problem by tiling the signal output of the different wavelets spatially in the signal 
window. Because a translation in the spatial domain results in a linear phase shift in the frequency domain of the DEs we only have to add linear phases to the corresponding wavelet filter functions and superpose them. Taking a two dimensional version of the continuous wavelet transform (1) results in an impulse response

$$
W(\mathbf{x})=\sum_{j=1}^{n} \psi_{j}(\mathbf{x}) * \delta\left(\mathbf{x} \Leftrightarrow \mathbf{t}_{\mathbf{j}}\right)
$$

which leads to a filter function

$$
\widehat{W}(\mathbf{u})=\sum_{j=1}^{n} \widehat{\psi}_{j}(\mathbf{u}) e^{2 \pi i \mathbf{u t}_{\mathbf{j}}},
$$

in the plane of the DE, where $\mathbf{t}_{\mathbf{j}}$ stands for the translation vector of the different tiles in the signal window.

In the following example we use the mexican hat wavelet, defined in section 2 , with four different scales $a=8,4,2, \sqrt{2}$, which can immediately be seen in the rightmost image from the upper left to the bottom right. Also in the amplitude image of the complex filter the superposition of the four scaled mexican hats are visible as rings with different diameters. This filter function or rather its Fourier transform serves as the initial point $h_{0}$ for the calculation of the phase-only DE shown in the middle of figure 4. Remark that edges contained in the input signal transforms to nullstellen in the output signal.
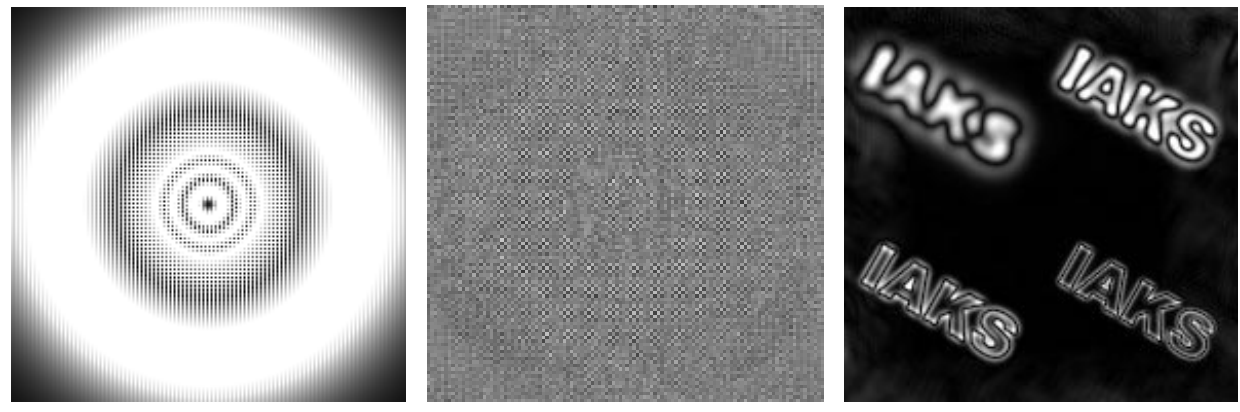

Figure 4. Left: Amplitude of complex filter. Center: Calculated phaseonly DE. Right: Output signal window.

The calculated DE is producable using standard techniques, e.g. with lithographic methods. Using spatial light modulators (SLM) is another, 
more flexible way to realize the DE, because of the dynamical optical or electronical addressing possibilities of SLMs.

\section{Conclusion}

Wavelets are a flexible language to describe signals. We sketched how they can be used for an efficient signal representation in compression applications. So wavelet bases can be interpreted as "universal dictionary" for signal and image description. If we restrict our interest to special features of the images it may be adequate to use a more expressive "specialized dictionary".

We described an optical implementation of the wavelet transform that allows an extremely fast image preprocessing. This implementation is suitable for pattern recognition applications such as texture analysis or feature extraction. Intelligent medical imaging systems require more elaborated non-linear image processing transforms. This can be achieved with a combination of optical and traditional computation technologies. Some promising experiments with such an hybrid opto-electronical setup have been performed at the authors' institution [2].

\section{References}

1. H. Aagedal, Th. Beth, J. Müller-Quade, and M. Schmid. Algorithmic design of diffractive optical systems for information processing. Submitted to: PhysComp96, Boston, November 1996.

2. H. Aagedal, Th. Beth, H. Schwarzer, and S. Teiwes. Modern concepts for computeraided design in diffractive optics. In G. W. Forbes, editor, OSA Proceedings of the International Optical Design Conference, volume 22, pages 257-260. Optical Society of America, Washington DC), 1994.

3. R. Barakat and G. Newsam. Algorithms for reconstruction of partially known, bandlimited fourier-transform pairs from noisy data. Journal of the Optical Society of America, 2:2027-2039, 1985.

4. R. W. Gerchberg and W. O. Saxton. A practical algorithm for the determination of phase from image and diffraction plane pictures. Optik, 35:237-246, 1972.

5. L. G. Gubin, B. T. Poljak, and E. V. Raik. The method of projections for finding the common point of convex sets. USSR Computational Mathematics and Mathematical Physics, 3(6):1-24, 1967.

6. J. P. Kahane and P.-G. Lemarié-Rieusset. Fourier Series and Wavelets, volume 3 of Studies in the Development of Modern Mathematics. Gordon and Breach Publishers, 1995.

7. A. Levi and H. Stark. Image restoration by the method of generalized projections with application to restoration from magnitude. Journal of the Optical Society of America, 1:932-943, 1984.

8. A. K. Louis, P. Maaß, and A. Rieder. Wavelets. Teubner, 1994. 
9. F. May, A. Klappenecker, V. Baumgarte, A. Nückel, and T. Beth. A high throughput multiplication free approximation to arithmetic coding. To appear in: Proceedings 1996 International Symposium on Information Theory and Its Applications, 1996.

10. F. Wyrowski and O. Bryngdahl. Digital holography as part of diffractive optics. Reports on Progress in Physics, 54:1481-1571, 1991. 